\title{
Spin identification of the RS resonance in di-photon production to NLO at the LHC
}

\author{
M. C. Kumar \\ Deutsches Elektronen-Synchrotron DESY, Platanenallee 6, D-15738 Zeuthen, Germany \\ E-mail: kumar.meduriedesy.de
}

Prakash Mathews*

Saha Institute of Nuclear Physics, 1/AF Bidhan Nagar, Kolkata 700064, India

E-mail: prakash.mathews@saha.ac.in

\section{A. A. Pankov}

The Abdus Salam ICTP Affiliated Centre, Technical University of Gomel, 246746 Gomel, Belarus

E-mail: pankov@ictp.it

\section{N. Paver}

University of Trieste and INFN-Trieste Section, 34100 Trieste, Italy

E-mail: nello.paverets.infn.it

\section{Ravindran}

Regional Centre for Accelerator-based Particle Physics

Harish-Chandra Research Institute, Chhatnag Road, Jhunsi, Allahabad 211 019, India

E-mail: ravindra@hri.res.in

\section{A. V. Tsytrinov}

The Abdus Salam ICTP Affiliated Centre, Technical University of Gomel, 246746 Gomel, Belarus

E-mail: tsytrin@rambler.ru

\begin{abstract}
We study the identification of a spin-2 Randall-Sundrum graviton resonance against the hypothesis of a gauge singlet spin- 0 scalar exchange giving the same number of events under the peak, by means of the symmetrically integrated angular asymmetry $A_{C E}$, evaluated for both graviton and scalar s-channel exchanges to next-to-leading order in QCD.
\end{abstract}

Loops and Legs in Quantum Field Theory - 11th DESY Workshop on Elementary Particle Physics, April 15-20, 2012

Wernigerode, Germany

\footnotetext{
* Speaker.
} 


\section{Introduction}

The diphoton production at the LHC is an important channel to study the physics beyond the standard model (SM). Extensions of the SM, predict particles of different spin, that could be produced at the LHC. These resonances would subsequently decay to SM particles and the angular distribution contains information about the coupling and spin etc., of the resonance. One such model is the warped extra dimension model (RS) [1] which postulates the existence of massive Kaluza-Klein (KK) modes. In addition to the SM diphoton, the spin-2 RS resonance could also decay to two photons. Now it is essential to distinguish the diphoton as a result of a spin-2 decay from other resonances, which could only be due to a spin-0 scalar resonance.

The interaction Lagrangian of the spin-2, RS KK mode $h_{\mu \nu}^{(n)}$ as a result of the graviton propagating the warped extra dimensions with the SM fields constrained on the TeV brane, is via the energy momentum tensor $T^{\mu v}$ of the SM

$$
\mathscr{L}_{G}=-\frac{1}{\Lambda_{\pi}} T^{\mu v} \sum_{n=1}^{\infty} h_{\mu v}^{(n)}
$$

where $\Lambda_{\pi}$ of the order of a TeV is the effective mass scale of the RS model. The characteristic mass spectrum of the KK mode is $M_{n}=x_{n} k \exp \left(-k \pi R_{c}\right)$ (with $x_{n}$ the roots of the Bessel function $J_{1}\left(x_{n}\right)=0, k$ the 5-d curvature assumed to be of the order of Planck mass $M_{P l}$ and $R_{c}$ is the radius of compactification). The model can therefore conveniently be parameterized in terms of $M_{G} \equiv M_{1}$, the mass of the lowest graviton excitation, and of the universal dimensionless graviton coupling $c=k / \bar{M}_{\mathrm{Pl}}$.

For the scalar particle $S$, we consider the simple model [2], singlet under the SM gauge group and with mass $M_{S}$, of the TeV order. The couplings of $S$ with gluons $\left(G_{\mu \nu}^{a}\right)$, electroweak gauge bosons $\left(W_{\mu \nu}^{i}\right)$ and fermions $(f)$, are given by:

$$
\mathscr{L}_{S}=c_{3} \frac{g_{s}^{2}}{\Lambda} G_{\mu \nu}^{a} G^{a \mu v} S+c_{2} \frac{g^{2}}{\Lambda} W_{\mu \nu}^{i} W^{i \mu v} S+c_{1} \frac{g^{\prime 2}}{\Lambda} B_{\mu \nu} B^{\mu v} S+\sum_{f} c_{f} \frac{m_{f}}{\Lambda} \bar{f} f S
$$

where $\Lambda$ is a high mass scale, of order $\mathrm{TeV}$, and $c_{i}$ 's are dimensionless coefficients that are assumed to be of order unity, reminiscent of a strong novel interaction.

\section{Center-Edge Asymmetry}

The total cross section for a heavy resonance discovery in the events at a diphoton invariant mass $M=M_{R}$ can be expressed as

$$
\sigma(p p \rightarrow \gamma \gamma)=\int_{-z_{\text {cut }}}^{z_{\text {cut }}} \mathrm{d} z \int_{M_{R}-\Delta M / 2}^{M_{R}+\Delta M / 2} \mathrm{~d} M \frac{\mathrm{d} \sigma}{\mathrm{d} M \mathrm{~d} z}
$$

where the rapidity of the individual photon $\left|\eta_{\gamma}\right|<2.5$ and $z=\cos \theta$ is chosen such that $|z|<$ 0.98. Resonance spin-diagnosis uses the comparison between the characteristic photon differential distributions for the two hypotheses for the resonance bump, $R=G$ spin-2 KK modes of the RS graviton and $R=S$ a massive scalar:

$$
\frac{\mathrm{d} \sigma}{\mathrm{d} z}=\int_{M_{R}-\Delta M / 2}^{M_{R}+\Delta M / 2} \mathrm{~d} M \frac{\mathrm{d} \sigma}{\mathrm{d} M \mathrm{~d} z} .
$$



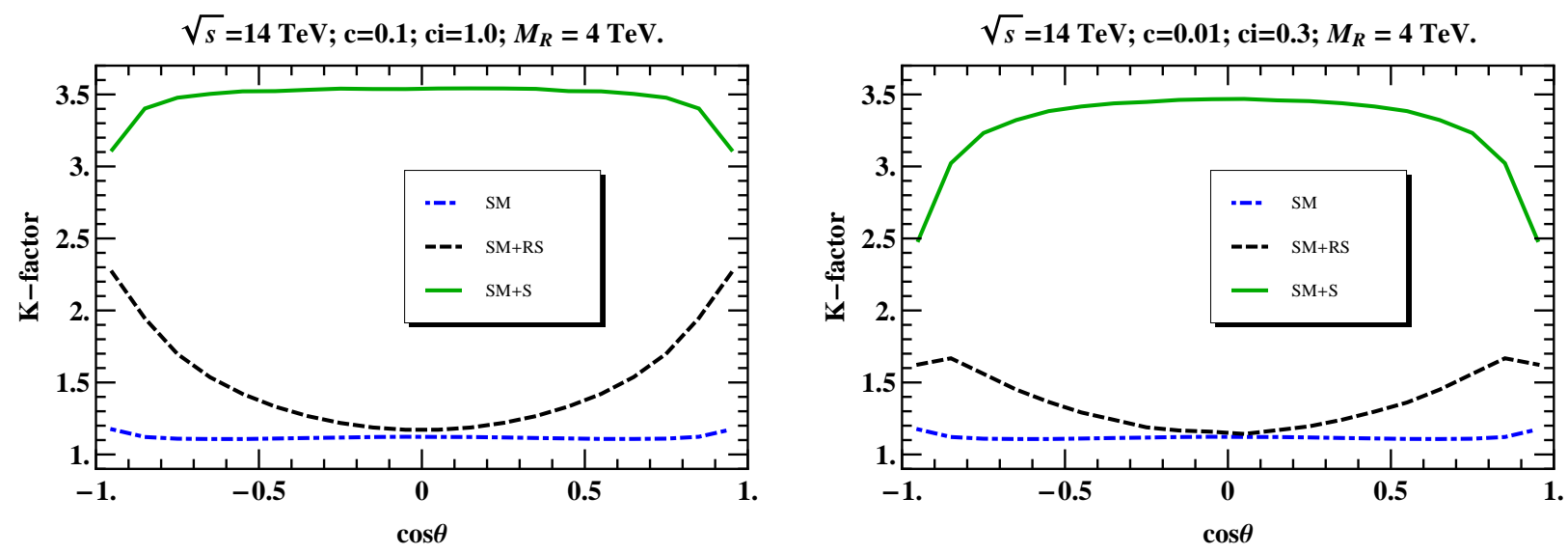

Figure 1: K-factor $v s \cos \theta$ for the $14 \mathrm{TeV}$ LHC.

In Eqs. (2.1) and (2.2), cuts on the phase space accounting for detector acceptance are implicit, and $\Delta M$ is an invariant mass bin around $M_{R}$, which should somehow reflect the detector energy resolution and be sufficiently large as to include the resonance width. The $z$-evenly-integrated center-edge angular asymmetry $A_{\mathrm{CE}}$ is defined as [3]:

$$
A_{\mathrm{CE}}=\frac{\sigma_{\mathrm{CE}}}{\sigma} \quad \text { with } \quad \sigma_{\mathrm{CE}} \equiv\left[\int_{-z^{*}}^{z^{*}}-\left(\int_{-z_{\mathrm{cut}}}^{-z^{*}}+\int_{z^{*}}^{z_{\mathrm{cut}}}\right)\right] \frac{\mathrm{d} \sigma}{\mathrm{d} z} \mathrm{~d} z
$$

where, $0<z^{*}<z_{\text {cut }}$ defines the separation between the "center" $\left(|z|<z^{*}\right)$ and the "edge" $\left(z^{*}<\right.$ $\left.|z|<z_{\text {cut }}\right)$ angular regions and is a priori arbitrary, but from previous experience the "optimal" numerical value we chose is $z^{*} \simeq 0.5$.

\section{Next-to-leading order QCD effects}

The diphotons in the SM to LO in QCD are produced in the quark anti-quark annihilation subprocess $q \bar{q} \rightarrow \gamma \gamma$. Photon pairs produced via the gluon fusion subprocess through a quark box diagram $g g \rightarrow \gamma \gamma$, though of the order $\alpha_{s}^{2}$, have cross sections comparable to those of the $q \bar{q} \rightarrow \gamma \gamma$ subprocess for the low diphoton invariant mass. In the light Higgs boson mass region, this subprocess plays an important role, due to the large gluon flux at small fractional momentum $x$, and is treated as a LO contribution though it is in reality a next-to-next-to leading order contribution. However, it falls off rapidly with increasing diphoton invariant mass and in the mass range of interest for the TeV scale gravity models it is not included at LO [4]. Both the $q \bar{q}$ and $g g$ channel would contribute to the diphoton signal at LO via the s-channel exchange of a spin-2 KK mode $(\mathrm{G})$ or a scalar (S) resonance as a result of the new physics (NP) models.

The NLO QCD corrections to the diphoton production process would involve real emission diagrams corresponding to the following $2 \rightarrow 3$ subprocesses: (a) $q \bar{q} \rightarrow \gamma \gamma g$, (b) $g g \rightarrow \gamma \gamma g$ and (c) $g q(\bar{q}) \rightarrow \gamma \gamma q(\bar{q})$. In the virtual part, $\mathscr{O}\left(\alpha_{s}\right)$ corrections at one loop arise as a result of the interference between the one loop graphs at $\mathscr{O}\left(\alpha_{s}\right)$ of $(S M+N P)$ and the Born graphs at $\mathscr{O}\left(\alpha_{s}^{0}\right)$ of $(S M+N P)$. The $q \bar{q}$ channel gets such contributions from both the SM and the graviton exchange. In the $g g$ channel the SM contribution begins only at $\mathscr{O}\left(\alpha_{s}\right)$. The SM $g g \rightarrow \gamma \gamma$ subprocess 
amplitude can interfere with the gluon initiated LO gravity initiated subprocess, giving an $\mathscr{O}\left(\alpha_{s}\right)$ contribution. In the $q g$ channel there is no virtual contribution to this order in either the SM or the NP models of interest. The poles in $\varepsilon$ arise from loop integrals, corresponding to the soft and collinear divergences. Configurations where a virtual gluon momentum goes to zero corresponds to soft singularities while the collinear singularities arise when two massless partons become collinear to each other. The virtual contribution here does not contain UV singularities.

At higher orders in QCD, there would be final state collinear singularities of QED origin as a result of the emission of a photon from a quark. These singularities can be factored out and absorbed into the fragmentation functions. The fragmentation functions are additional non perturbative inputs that are not well-understood. An alternate approach to isolate direct photons is the smooth cone isolation criterion [5], which ensures that the fragmentation contributions are suppressed without affecting the cancellation of the conventional QCD singularities.

To deal with the various singularities of soft and collinear origin that appear in the corresponding real diagram to NLO, we use the semi-analytical two-cutoff phase space slicing method [6]. The 3-body phase space of the real emission diagrams has regions which are soft and collinear divergent. The phase space can accordingly be separated into soft $(s)$ and hard regions and, furthermore, the hard region can be separated into hard collinear $(H C)$ and hard non-collinear $(\overline{H C})$ parts as follows:

$$
d \sigma^{\text {real }}=d \sigma_{s}^{\text {real }}\left(\delta_{s}\right)+d \sigma_{H C}^{\text {real }}\left(\delta_{s}, \delta_{c}\right)+d \sigma_{\overline{H C}}^{\text {real }}\left(\delta_{s}, \delta_{c}\right) .
$$

The small cut-off parameters $\delta_{s}$ and $\delta_{c}$ set arbitrary boundaries for the soft (gluon energy $0 \leq$ $\left.E_{g} \leq \delta_{s} \sqrt{\hat{s}} / 2\right)$ and collinear $\left(0 \leq \hat{t}_{i j} \leq \delta_{c} \hat{s}\right)$ regions, respectively. Here, for the $2 \rightarrow 3$ process with momenta $p_{1}+p_{2}=p_{3}+p_{4}+p_{5}$, the Mandelstam variables are defined as $\hat{s}_{i j}=\left(p_{i}+p_{j}\right)^{2}$, $\hat{t}_{i j}=\left(p_{i}-p_{j}\right)^{2}$, with $\hat{s}=\hat{s}_{12}$. In these mutually exclusive soft and hard collinear regions, the 3body cross section can be factored into the Born cross section $(2 \rightarrow 2)$ and the remaining phase space integral can easily be evaluated in $(4+\varepsilon)$-dimensions to get the expansion of the soft and collinear singularities in powers of $\varepsilon$. All positive powers of the small cut-off parameters $\delta_{s}$ and $\delta_{c}$ are set to zero, only logarithms of the cut-off parameters are retained. Adding the virtual and real contributions, all double and single poles of IR origin are automatically cancelled between the virtual and the first two terms of Eq. (3.1). The remaining collinear poles are absorbed into the redefinition of the parton distribution functions.

The hard non-collinear part in Eq. (3.1) corresponds to the 3-body phase space, which by construction is finite, and can be evaluated in 4-dimensions $(\varepsilon=0)$. The sum of real and virtual contributions is now free of singularities and can hence be evaluated numerically using a MonteCarlo method. It can be further seen that the explicit logarithmic dependence on $\delta_{s}$ and $\delta_{c}$ in the 2-body phase space $\left(d \sigma_{s}^{\text {real }}\left(\delta_{s}\right)+d \sigma_{H C}^{\text {real }}\left(\delta_{s}, \delta_{c}\right)\right)$ is cancelled by the implicit dependence of the 3body hard non-collinear part $d \sigma_{H C}^{r e a l}\left(\delta_{s}, \delta_{c}\right)$, after the numerical integration is carried out. The sum of the 2-body and 3-body parts in Eq. (3.1) would be independent of the slicing parameters $\delta_{s}$ and $\delta_{c}$, which is explicitly verified before the code is used for the analysis.

In the scalar model, the UV divergences in the virtual corrections to the $g g$ initiated diphoton production process are removed by renormalization. The remaining finite contribution is given by

$$
\frac{d \sigma_{v}}{d z}=\frac{g_{s}^{2}}{16 \pi^{2}} 8 N \zeta(2) \frac{d \sigma}{d z},
$$




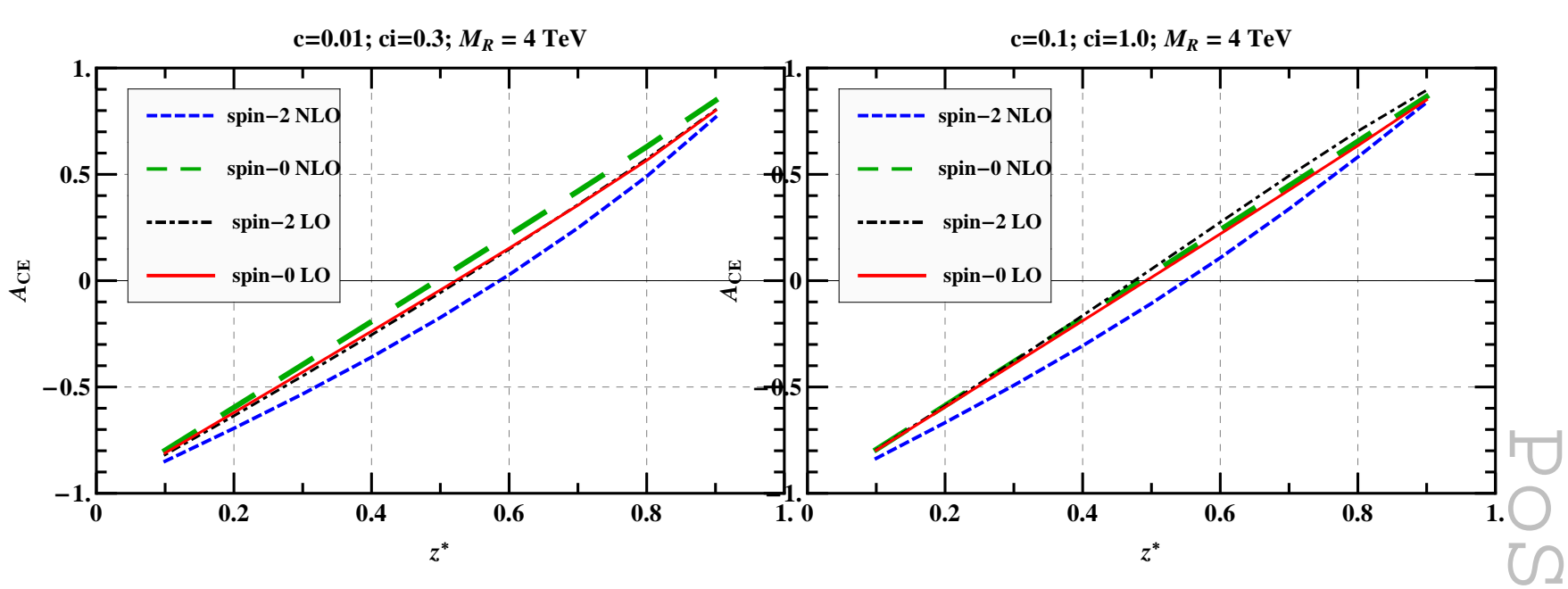

Figure 2: NLO $A_{\mathrm{CE}}$ asymmetry vs $z^{*}$ at the $14 \mathrm{TeV}$ LHC for the RS model with graviton coupling constant $c=0.01(0.1)$, and the scalar resonance model with $c_{3}=0.3, c_{i}=0.3\left(c_{3}=0.3, c_{i}=1.0\right)$ and $M_{R}=4 \mathrm{TeV}$.

where $d \sigma / d z$ is the Born contribution [7]. The remaining soft and collinear finite contributions coming from the real corrections to the LO scalar model diagram are proportional to the Born cross section. As they originate from pure QCD, they are independent of the hard scattering process, hence they will be the same as those for the $s$-channel diphoton production process in the RS model and are given in Ref. [4].

At the NLO in QCD, the following three subprocesses contribute to the scalar model cross section: (i) $\bar{q} q \rightarrow g S$ (ii) $q g \rightarrow q S$ and (iii) $g g \rightarrow g S$, all followed by $S \rightarrow \gamma \gamma$. The Feynman diagrams for the $\bar{q} q$ and $g g$ channels are given in [7]. The couplings of $S$ to the light quarks are proportional to the masses and hence are negligible, so the main contribution to the $\bar{q} q$ channel is from the $S$ coupling to the gluons. Again the $q g$ channel is related to the $\bar{q} q$ channel by suitable change of initial and final state.

The K-factor as a function of the $\cos \theta$ for SM, spin-2 and scalar case has been plotted in Fig. 1 for typical parameters of couplings at the $14 \mathrm{TeV}$ LHC. The K-factor represent the quantitative magnitude of the NLO QCD corrections and could be large. Including these higher order QCD corrections to an observable at the hadron collider reduces the dependence on the factorization and renormalization scales.

For the diphoton resonance with an invariant mass of $4 \mathrm{TeV}$ at the $14 \mathrm{TeV} \mathrm{LHC}$, we have plotted the $A_{C E}$ as a function of $z^{*}$ for both LO and NLO for the spin 2 and scalar resonance in Fig. 2. An important feature of the NLO corrections is that, for the scalar exchange, $A_{C E}$ is practically unaffected, the K-factor remaining almost flat in $\cos \theta$ whereas this is not so for the spin- 2 case (Fig. 1). The NLO effects tend to enlarge the difference between the $A_{C E}$ relevant to the two cases, indicating a better possibility of distinguishing the models by the measurement of this observable.

Finally, in Fig. 3 we show the RS graviton signature domain for $0.01 \leq c \leq 0.1$ at LO (left panel) and to NLO (right panel) in QCD. The minimum number of events statistically needed for discovering the first RS KK mode in the diphoton channel at 5- $\sigma$ level is indicated by the red line labelled "Discovery". For different values of $M_{G}$ the lines marked with $c$ (RS KK mode) and $c_{i}$ (scalar), denote the events for diphoton production as a result of the decay of RS KK mode and 

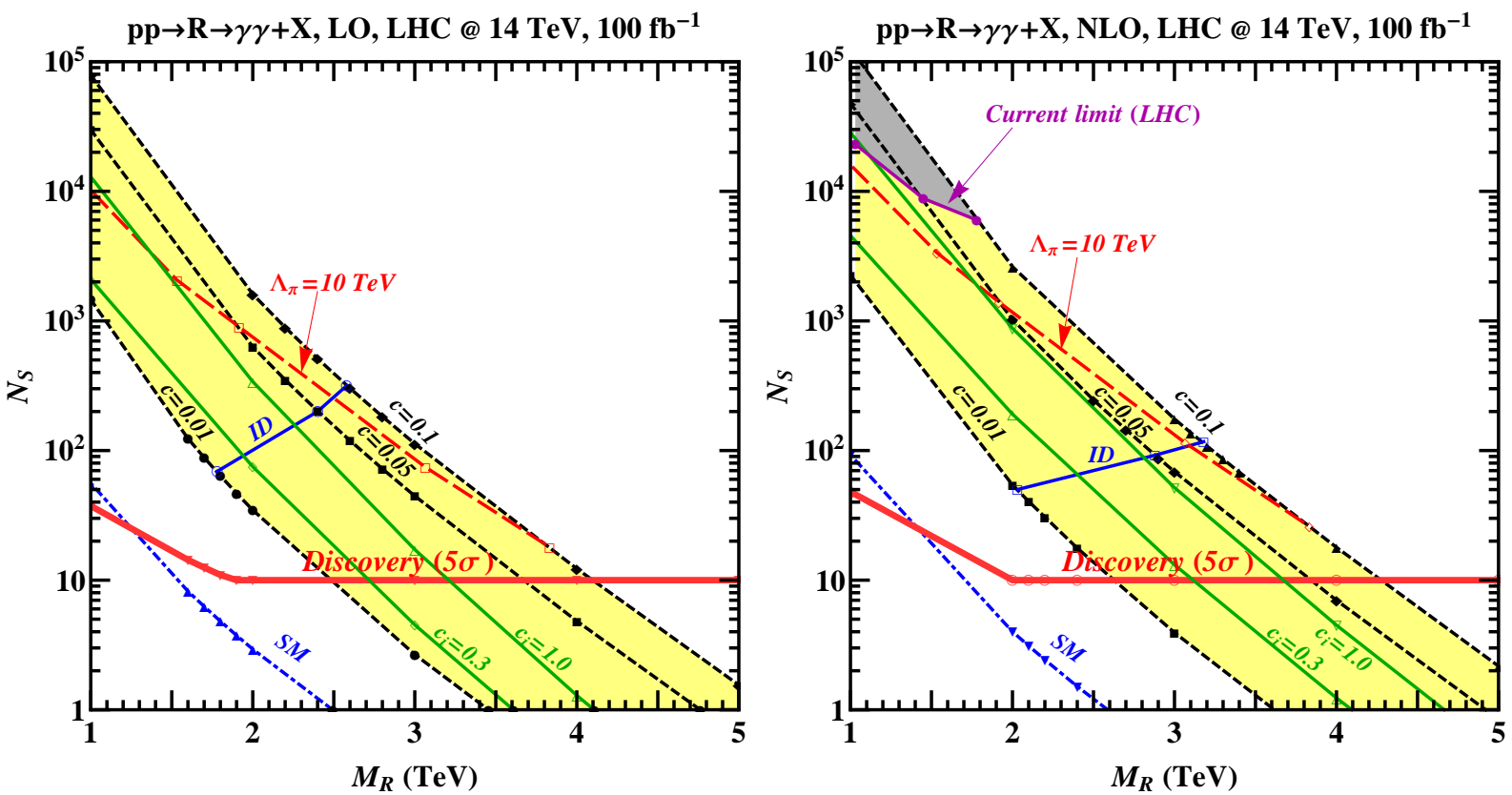

Figure 3: Number of resonance (signal) events $N_{S}$ vs $M_{R}(R=G, S)$ at the LHC with $\sqrt{s}=14 \mathrm{TeV}$ and $\mathscr{L}_{\text {int }}=100 \mathrm{fb}^{-1}$ for the process $p p \rightarrow G \rightarrow \gamma \gamma+X$ at LO (left panel) and NLO (right panel) QCD. The yellow area corresponds to the KK graviton signature space for $0.01 \leq c \leq 0.1$; the signature space for the scalar $S$ resonance is represented by the two specific cases, $c_{3}=c_{i}=0.3$ and $c_{3}=0.3, c_{i}=1.0$.

scalar respectively. The line labeled "ID" refers to the minimum number of events needed for a discovered RS graviton resonance to be identified at 95\% CL against the scalar particle exchange hypothesis.

The NLO QCD computation of the $A_{C E}$ angular analysis, modifies the identification limits of a spin-2 KK mode of the RS resonance in the range $2-3 \mathrm{TeV}$ for $c=0.01-0.1$, in comparison to a spin- 0 hypothesis at the $14 \mathrm{TeV}$ LHC with $100 \mathrm{fb}^{-1}$ of luminosity.

\section{References}

[1] L. Randall and R. Sundrum, Phys. Rev. Lett. 83, 3370 (1999) [arXiv:hep-ph/9905221].

[2] R. Barbieri and R. Torre, Phys. Lett. B 695, 259 (2011) [arXiv:1008.5302 [hep-ph]].

[3] P. Osland, A. A. Pankov, N. Paver and A. V. Tsytrinov, Phys. Rev. D 78, 035008 (2008) [arXiv:0805.2734 [hep-ph]].

[4] M. C. Kumar, P. Mathews, V. Ravindran and A. Tripathi, Nucl. Phys. B 818, 28 (2009) [arXiv:0902.4894 [hep-ph]]; M. C. Kumar, P. Mathews, V. Ravindran and A. Tripathi, Phys. Lett. B 672, 45 (2009) [arXiv:0811.1670 [hep-ph]].

[5] S. Frixione, Phys. Lett. B 429, 369 (1998) [arXiv:hep-ph/9801442].

[6] B. W. Harris and J. F. Owens, Phys. Rev. D 65, 094032 (2002) [arXiv:hep-ph/0102128].

[7] M.C. Kumar, P. Mathews, A.A. Pankov, N. Paver, V. Ravindran and A.V. Tsytrinov, Phys. Rev. D84, 115008 [arXiv:1108.3764 [hep-ph]. 